\title{
Trends in Aerobic and Muscle-Strengthening Physical Activity by Race/Ethnicity Across Income Levels Among US Adults, 1998-2018
}

\author{
Kathleen B. Watson, Geoffrey Whitfield, Tiffany J. Chen, Eric T. Hyde, and John D. Omura
}

\begin{abstract}
Background: Although disparities in leisure-time physical activity (LTPA) participation by race/ethnicity and income are known, the combined association of these characteristics with LTPA participation is less understood. This study aims to describe trends and determine whether racial/ethnic differences in adult physical activity by income level have changed over the past 2 decades. Methods: The authors estimated LTPA participation (outcomes: any aerobic activity, meeting the aerobic activity guideline, meeting the muscle-strengthening guideline, and meeting the combined aerobic and muscle-strengthening guidelines) among adults $\geq 18$ years by race/ethnicity across income levels using 1998-2018 National Health Interview Survey data in 3-year aggregates. They also tested for trends, prevalence differences, and difference in differences using logistic regression. Results: LTPA participation increased from 1998-2000 to 2016-2018 for all outcomes for non-Hispanic white, non-Hispanic black, and Hispanic adults at all income levels. Disparities narrowed for some groups but persisted between white and racial/ethnic minority groups across income levels for engaging in any aerobic activity and meeting the aerobic guideline $(0.2-8.8$ percentage point difference in differences). Disparities in meeting the muscle-strengthening and combined guidelines were less common. Conclusions: Opportunities exist to ensure that adults, particularly members of lower income racial/ethnic minority groups, have support to help them participate in LTPA.
\end{abstract}

Keywords: surveillance, epidemiology, equity

Regular physical activity provides substantial health benefits. ${ }^{1,2}$ Current federal guidelines recommend that adults move more and sit less throughout the day; adults who sit less and do any amount of moderate to vigorous physical activity gain some health benefits. ${ }^{2}$ For substantial health benefits, adults should do at least 150 minutes a week of moderate-intensity aerobic physical activity, or 75 minutes a week of vigorous-intensity aerobic physical activity, or an equivalent combination of moderate- and vigorous-intensity aerobic activity. ${ }^{2}$ Adults should also do musclestrengthening activities of moderate or greater intensity and that involve all major muscle groups on 2 or more days a week as these activities provide additional health benefits. ${ }^{2}$

Unfortunately, less than one-fourth of adults $(24.0 \%)$ meet the $\mathrm{Q}$ guidelines for both 1 leisure-time aerobic and muscle-strengthening physical activity. ${ }^{3}$ Moreover, physical activity levels consistently vary by certain demographic characteristics, including race/ ethnicity and income level. For example, physical activity participation is lower among black and Hispanic racial/ethnic minority groups compared with white adults ${ }^{4-9}$ and among adults from lower income households compared with adults from higher income households. ${ }^{9}$ Black and Hispanic adults also experience higher rates of chronic diseases, including diseases that physical activity helps prevent (eg, cardiovascular disease, obesity, type 2 diabetes, some cancers). ${ }^{1,2,10,11}$ Although disparities in physical activity participation by racial/ethnic groups and income levels are well established, the combined influence of race/ethnicity and

The authors are with the Division of Nutrition, Physical Activity, and Obesity, National Center for Chronic Disease Prevention and Health Promotion, Centers for Disease Control and Prevention, Atlanta, GA, USA. Watson (iyr4@cdc.gov) is corresponding author. income level on physical activity participation has been less studied.

Differences in social determinants of health contribute to these disparities. Social determinants of health are the conditions in the environments where people are born, live, learn, work, play, worship, and age ${ }^{12}$ and include factors associated with physical activity, such as neighborhood environment, safety, and income. ${ }^{12}$ Compared with non-Hispanic whites, some barriers to physical activity participation are reported more often among black and Hispanic adults. For example, in 2015, non-Hispanic blacks and Hispanics reported crime and animals as barriers to safe walking more frequently than non-Hispanic whites. ${ }^{13}$ US Census data show that blacks and Hispanics have the highest poverty rates compared with other racial/ethnic groups, ${ }^{14}$ and poverty has been associated with physical activity participation. ${ }^{15}$ In a study among public housing residents, blacks reported that opportunities for being active were limited and not readily available and that the neighborhood environment was being neglected (eg, sidewalks in disrepair). ${ }^{14,16}$ Understanding whether measures of socioeconomic status, such as income, explain difference in physical activity behavior by race/ethnicity is important to efforts to eliminate health disparities.

Monitoring disparities in leisure-time physical activity (LTPA) participation can help health officials and policymakers plan, implement, and evaluate initiatives that promote physical activity, particularly among populations that would most benefit. Trends in the prevalence of meeting national guidelines for aerobic and muscle-strengthening activities (separately and combined) among US adults have been examined over the past 2 decades overall, by race/ethnicity, and by education level (as an indicator of socioeconomic status). ${ }^{3,17}$ Similar estimates are also monitored as part of the Healthy People initiative, which is the leading disease 
prevention and health promotion road map for the nation. ${ }^{18}$ Beyond these efforts, little attention has been paid to the combined influence of race/ethnicity and socioeconomic status on differences in physical activity levels over time. Because racial/ethnic minority groups tend to have higher rates of chronic disease and lower incomes, assessing trends in physical activity over time by race/ ethnicity and across income levels may help identify priority populations that would especially benefit from promotional efforts to increase physical activity.

The objectives of this study were to (1) describe trends in the prevalence of LTPA participation (as measured by participation in any aerobic physical activity, meeting the aerobic guideline, meeting the muscle-strengthening guideline, and meeting both the aerobic and muscle-strengthening guidelines) among US adults by race/ethnicity across income levels from 1998-2000 to 20162018, (2) determine whether differences in the prevalence of LTPA participation by racial/ethnic groups exist across income levels, and (3) determine whether differences in the prevalence of LTPA participation by racial/ethnic groups across income levels have changed over the past 2 decades.

\section{Methods}

\section{Survey and Analytic Sample}

The National Health Interview Survey (NHIS) is a continuous, cross-sectional survey of US households. ${ }^{19}$ NHIS uses a multistage area probability design to create a nationally representative sample of the noninstitutionalized, civilian population. It collects basic health and demographic information from all family members and additional data, such as information about LTPA, from one randomly selected household adult aged $\geq 18$ years. During 19982018, sample sizes ranged from 21,781 (2008) to 36,697 (2014) and response rates ranged from $53.0 \%$ (2017) to $74.3 \%$ (2002). ${ }^{19}$ Additional information about the NHIS survey is available elsewhere. ${ }^{19}$

Across all years, the sample for this study included data from 600,550 non-Hispanic white, non-Hispanic black, and Hispanic adult respondents aged $\geq 18$ years. Data from 35,030 respondents who reported non-Hispanic "other" race categories, including multiracial, were not included in the study because of small sample sizes. Adults who were missing information on physical activity $(n=15,480)$ or selected characteristics $(n=23,123)$ were excluded. The final analytic sample had data from the remaining 561,947 $(93.6 \%)$ respondents.

\section{Measures}

NHIS respondents were classified by sex, age group (18-24, 2534 , 35-44, 45-64, or $\geq 65 \mathrm{y}$ ), and race/ethnicity (non-Hispanic white [white], non-Hispanic black [black], or Hispanic). Income level was measured using a ratio of the family's income in relation to the appropriate federal poverty threshold (poverty income ratio, PIR). Although there are no standard thresholds for classifying PIR, some thresholds are used to receive financial assistance. For example, one criterion to be eligible for the Supplemental Nutrition Assistance Program is to have PIR at or below $1.3 .{ }^{20}$ Several thresholds are used to receive assistance with health insurance through a state marketplace or the Federal Health Insurance Exchange (PIR at or below 1.38 for coverage through Medicaid expansion and PIR at or below 4.0 to qualify for tax credits). ${ }^{21}$ For our study, we classified income level as low (PIR $<1.50)$, moderate $(1.50 \leq \mathrm{PIR}<4.00)$, or high $(\mathrm{PIR} \geq 4.00)$.

Aerobic physical activity was assessed with 2 questions that asked how often during leisure time the respondent participated in (1) vigorous-intensity activities that caused heavy sweating or large increases in breathing or heart rate and (2) light- or moderateintensity activities that caused only light sweating or slight to moderate increases in breathing or heart rate for $\geq 10$ minutes at a time. Respondents provided both frequency of their participation and duration of each session. Respondents self-defined the time frame (per month or per week). Total leisure-time moderateintensity equivalent physical activity was calculated as weekly minutes of light- or moderate-intensity physical activity plus twice the reported minutes of vigorous-intensity physical activity. ${ }^{2} \mathrm{Re}$ spondents were then classified as engaging in any aerobic physical activity if their total leisure-time moderate-intensity equivalent physical activity lasted more than 10 minutes (engaging in activity). They were classified as meeting the minimal aerobic guideline (aerobic activity guideline) if they reported $\geq 150$ minutes per week of moderate-intensity equivalent activity. ${ }^{2}$ Muscle-strengthening activity was assessed with one question about how often the respondent participated in leisure-time physical activities specifically designed to strengthen their muscles, such as lifting weights or doing calisthenics. Respondents were categorized as meeting the muscle-strengthening guideline if they reported participating in muscle-strengthening activities 2 or more times per week. ${ }^{2}$ Lastly, respondents who reported meeting both the aerobic activity guideline and the muscle-strengthening guideline were classified as meeting the combined aerobic activity and muscle-strengthening guidelines.

\section{Statistical Analyses}

To ensure robust sample sizes for all race/ethnicity and income stratifications, respondents were pooled into 3-year intervals: 1998-2000, 2001-2003, 2004-2006, 2007-2009, 2010-2012, 2013-2015, and 2016-2018. We calculated the predicted prevalence of the 4 physical activity outcomes (engaging in any aerobic activity, meeting the aerobic activity guideline, meeting the muscle-strengthening guideline, and meeting the combined aerobic activity and muscle-strengthening guidelines) by race/ethnicity across income groups over time using logistic regression adjusted for sex and age group. The logistic model included orthogonal polynomial contrasts to test for linear, quadratic, and cubic trends within each race/ethnicity and income group. The overall change (difference over time) in predicted prevalence for each race/ethnicity and income group was estimated by contrast statements that subtracted the prevalence in 1998-2000 from the prevalence in 2016-2018.

To determine whether differences by racial/ethnic and income groups existed in the prevalence of physical activity participation at the start and end of the study period, the difference in prevalence at the first time period (D1; 1998-2000) between white respondents and members of each racial/ethnic minority group for each income level (low, moderate, and high) was tested using contrast statements (Figure 1). This method was repeated for the last time period (D2; 2016-2018). To determine whether differences in prevalence narrowed or widened over time (difference in differences analysis), D2 was subtracted from the D1 (Figure 2). When the difference between white respondents and members of each racial/ethnic minority group by income level at the first time period was significantly larger than the difference at the second time period 

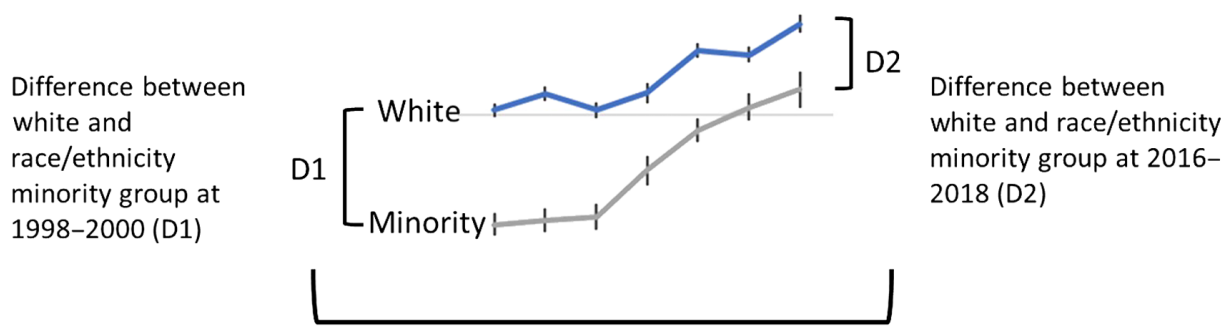

Change in difference [D1-D2] between white and racial/ethnic minority group ${ }^{a}$

Figure 1 - Description of the analyses to examine differences from 1998-2000 (D1) to 2016-2018 (D2) in the difference between white adults and members of racial/ethnic minority groups. Difference is narrowing (D1 - D2 $>0)$, difference remains unchanged (D1 $-\mathrm{D} 2=0)$, or difference is widening (D1 - D2 < 0). D1 indicates the difference in prevalence at the first time period (1998-2000); D2, difference in prevalence at the last time period (20162018). ${ }^{\mathrm{a}}$ Graphic depicts disparities with estimates for white $>$ minority group.

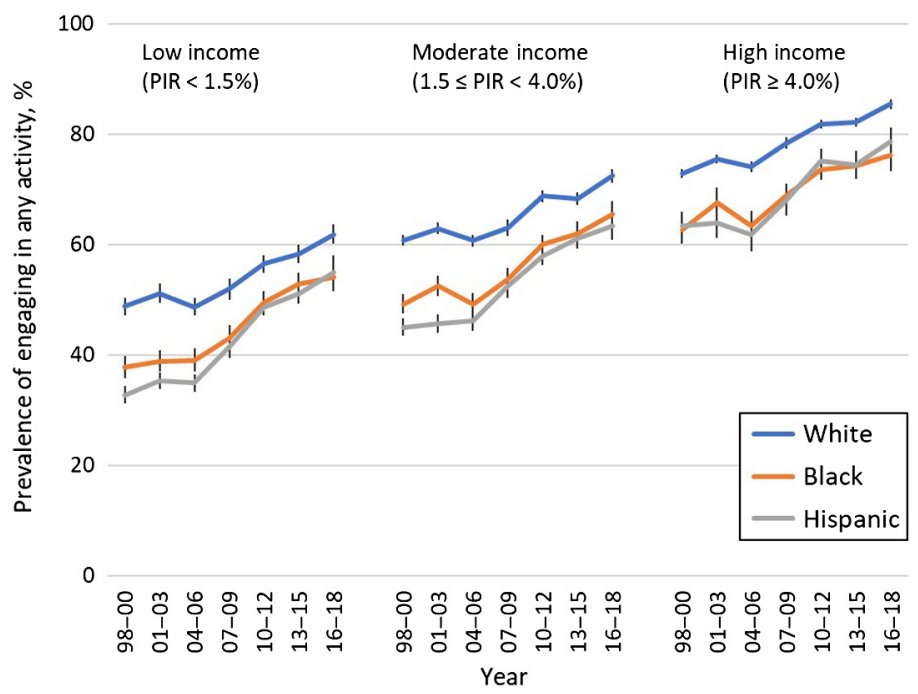

Figure 2 - Predicted marginals by 3-year intervals, racial/ethnic group, and income level for the prevalence of engaging in any aerobic activity among US adults, adjusted for age and sex. Error bars represent $95 \%$ confidence intervals. Participation in any aerobic activity was defined as engaging in any aerobic physical activity per week for $\geq 10$ minutes at a time. Black indicates non-Hispanic black; PIR, poverty income ratio; white, non-Hispanic white.

(D1 - D2 >0), the gap was defined as narrowing (Figure 2). When the difference at the first time period was significantly smaller than the second time period $(\mathrm{D} 1-\mathrm{D} 2<0)$, the gap was defined as widening. When there was no difference (D1 - D2 not significantly different from 0), the gap was defined as unchanged.

To handle high nonresponse rates for family income and personal earnings, the NHIS provided 5 multiple imputed data sets for income and earnings, as well as PIR, for each survey year. ${ }^{22}$ SAS-Callable SUDAAN (version 11.0; Research Triangle Institute, Research Triangle Park, NC) was used for all analyses to account for the complex sampling and multiple imputation design and to provide weighted estimates. Level of significance was $P \leq .05$. Bonferroni adjustments to $P<.008$ were used for multiple testing to control for inflated type I error.

\section{Results}

Across all racial/ethnic and income groups, the prevalence of all physical activity outcomes significantly increased from 1998-2000 to 2016-2018 (Table 1). Trends varied between linear only and linear, quadratic, and/or cubic trends, depending on racial/ethnic and income group and physical activity outcome (Table 1, Figures 2-5).

In 1998-2000, there were significant $(P<.008)$ differences between white and black adults in the prevalence of engaging in any aerobic activity and meeting the aerobic guideline across all income levels and in the prevalence of meeting the combined guidelines among low-income adults (Table 1). No differences in the prevalence of meeting the muscle-strengthening guideline were observed between white and black adults regardless of income level. Differences in the 1998-2000 prevalence between white and Hispanic adults were significant across all income levels and outcomes with 2 exceptions: no significant differences were observed in the prevalence of meeting the muscle-strengthening guideline or the combined guidelines among adults with high income (Table 1).

In 2016-2018, there were significant $(P<.008)$ differences between white and black adults and between white and Hispanic adults in the prevalence of engaging in any aerobic activity and meeting the aerobic guideline across all income levels (Table 1). Significant differences in the prevalence of meeting the musclestrengthening guideline and the combined guidelines were observed between white and Hispanic adults for low income and high income, respectively (Table 1).

Results for the difference in differences from 1998-2000 to 2016-2018 by racial/ethnic and income groups showed that differences in the prevalence estimates of engaging in any aerobic activity narrowed between white and black adults with moderate income (Figure 6). The difference in differences for the prevalence estimates of engaging in any aerobic activity and meeting the aerobic activity guideline narrowed between white and Hispanic adults with low and moderate income (Figure 6). Across all 3 income groups, there was a range of 0.2 to 8.8 percentage point difference in difference between white and Hispanic adults. Narrowing of the difference for meeting the muscle-strengthening guideline and the combined guidelines was limited to difference in difference between white 


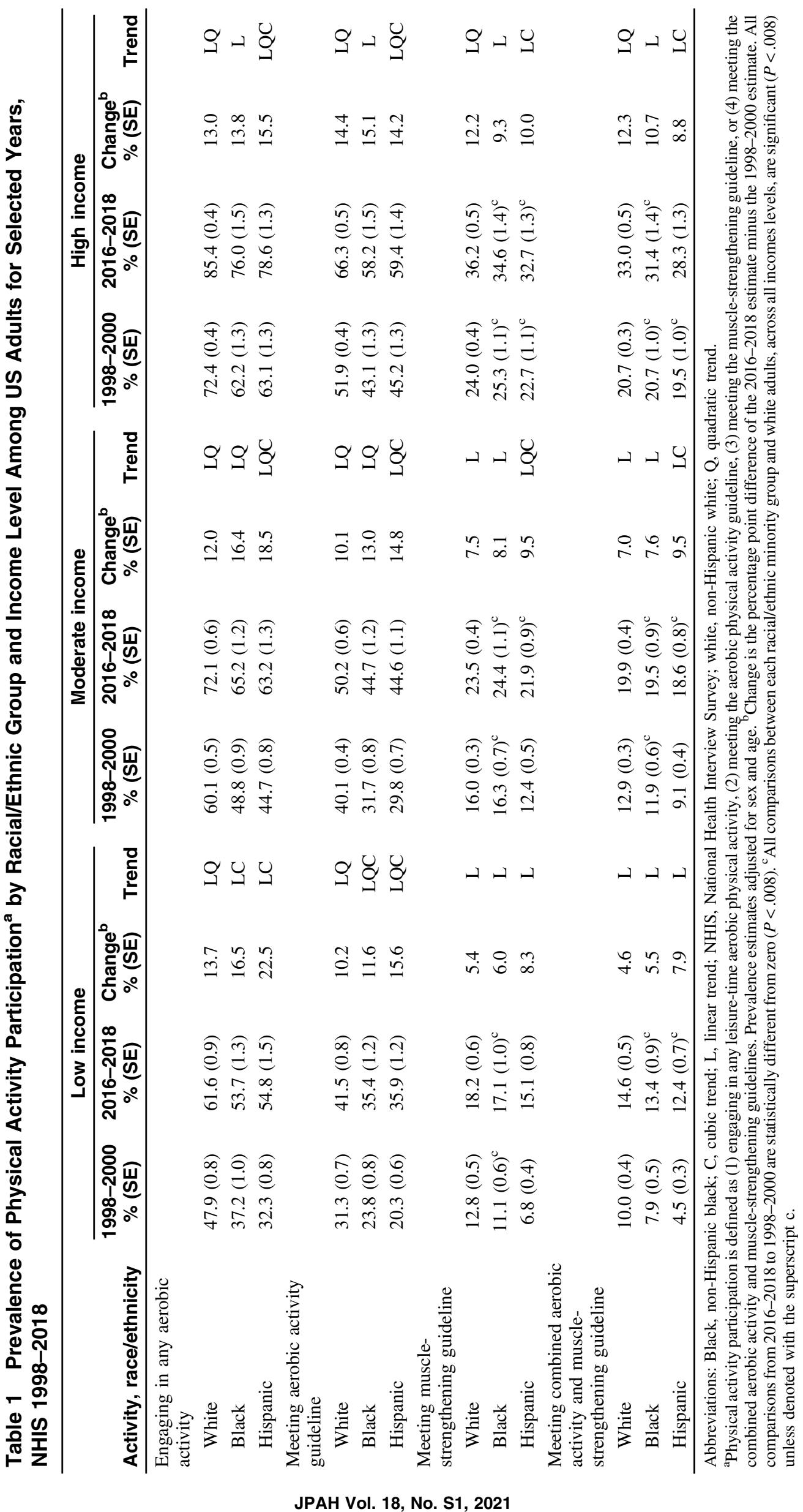




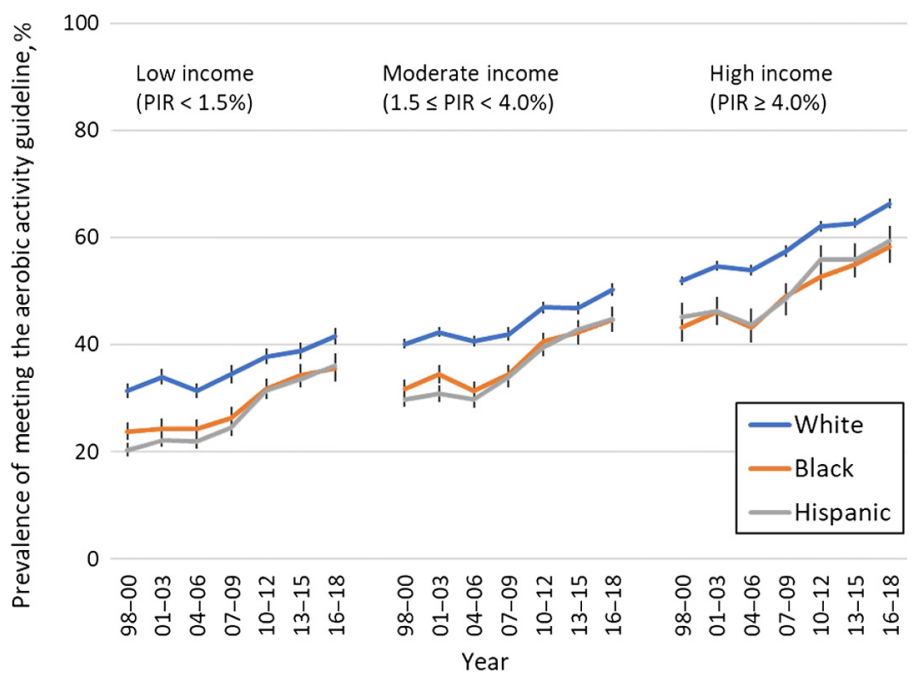

Figure 3 - Predicted marginals by 3-year intervals, racial/ethnic group, and income level for the prevalence of meeting the aerobic physical activity guideline (ie, engaging in $\geq 150 \mathrm{~min}$ of moderate-intensity equivalent aerobic activity per week) among US adults, adjusted for age and sex. Error bars represent $95 \%$ confidence intervals. Black or dashed lines indicates non-Hispanic black; PIR, poverty income ratio; white or solid line, non-Hispanic white.

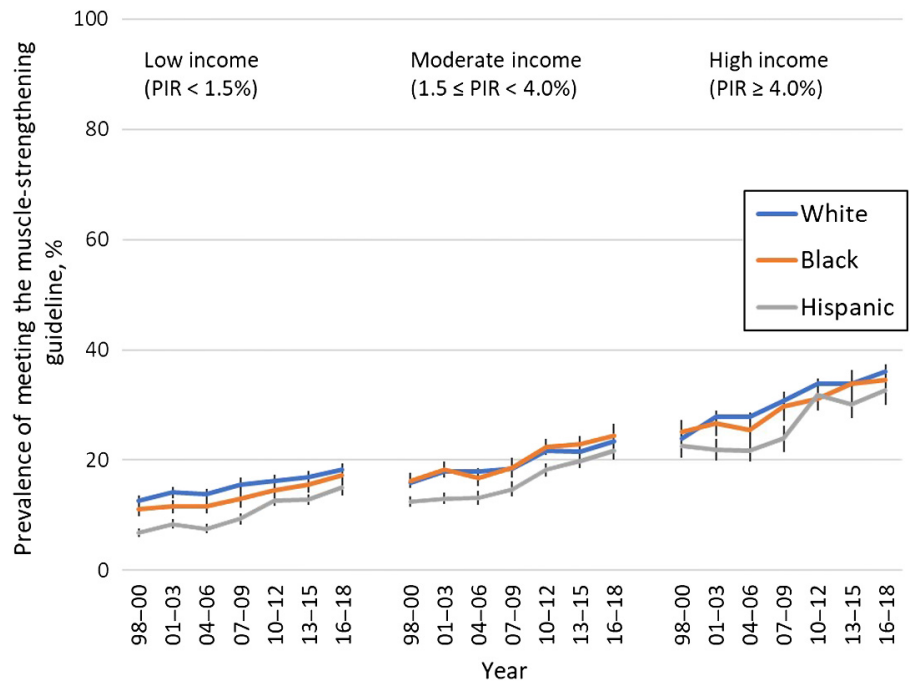

Figure 4 - Predicted marginals by 3-year intervals, race/ethnicity, and income for the prevalence of meeting the muscle-strengthening guideline (ie, engaging in muscle-strengthening activity on 2 or more days per week) among US adults, adjusted for age and sex. Error bars represent $95 \%$ confidence intervals. Black or dashed lines indicates non-Hispanic black; PIR, poverty income ratio; white or solid line, non-Hispanic white.

and Hispanic adults with low income (meeting the combined guidelines; $P<.008$; Figure 7).

\section{Discussion}

From 1998-2000 to 2016-2018, participation in physical activity, assessed using 4 measures, increased among white, black, and

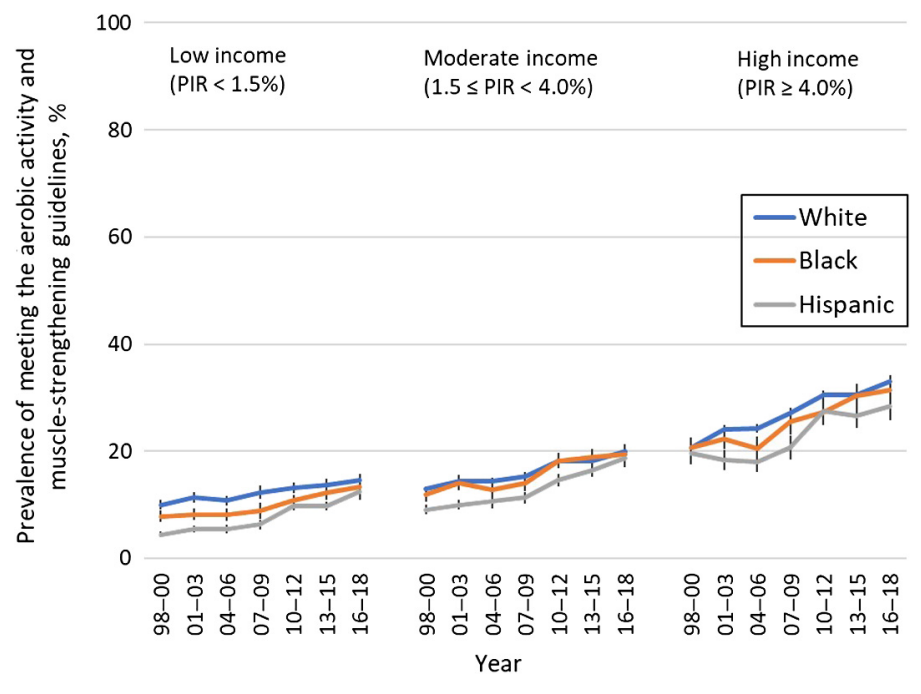

Figure 5 - Predicted marginals by 3 -year intervals, race/ethnicity, and income for the prevalence of meeting the combined aerobic activity and muscle-strengthening activity guidelines (ie, engaging in $\geq 150 \mathrm{~min}$ of moderate-intensity equivalent aerobic activity per week and engaging in muscle-strengthening activity on 2 or more days per week) among US adults, adjusted for age and sex. Error bars represent 95\% confidence intervals. Black or dashed lines indicates non-Hispanic black; PIR, poverty income ratio; white or solid line, non-Hispanic white.

Hispanic adults across all income levels. Despite these increases, disparities in the prevalence of engaging in any leisure-time aerobic activity and meeting the aerobic physical activity guideline persist between white populations and racial/ethnic minority groups across all income levels. Disparities are less frequently observed between white populations and racial/ethnic minority groups across income levels for meeting the muscle-strengthening guideline and for meeting the combined aerobic activity and musclestrengthening guidelines. These findings provide a more nuanced understanding of disparities in physical activity participation over time by race/ethnicity and income level. They can help identify specific populations that may especially benefit from efforts to promote physical activity.

National trends in physical activity by racial/ethnic groups and by income levels have been examined previously using different surveillance systems. Estimates from these systems showed that disparities in engaging in any leisure-time ${ }^{6}$ and nonoccupational ${ }^{8}$ physical activity persisted over time across racial/ethnic groups, with fewer black and Hispanic adults engaging in physical activity compared with white adults. In contrast, not all estimates for meeting the aerobic activity guideline were lower for black and Hispanic compared with white adults over time. Data from the National Health and Nutrition Examination Survey showed that disparities between white populations and racial/ethnic minority groups for meeting the aerobic activity guideline during leisure time narrowed from $2007-2008$ to $2017-2018 .{ }^{6}$ Education is an alternative indicator of socioeconomic status, and adult respondents in the National Health and Nutrition Examination Survey with higher educational attainment were more likely than adults from households with lower educational attainment to engage in any physical activity. ${ }^{6}$ Few studies have looked at physical activity levels stratified by racial/ethnic group and income level, so direct comparisons with the results from our study cannot be made. Regardless, the consistent improvement in physical activity 


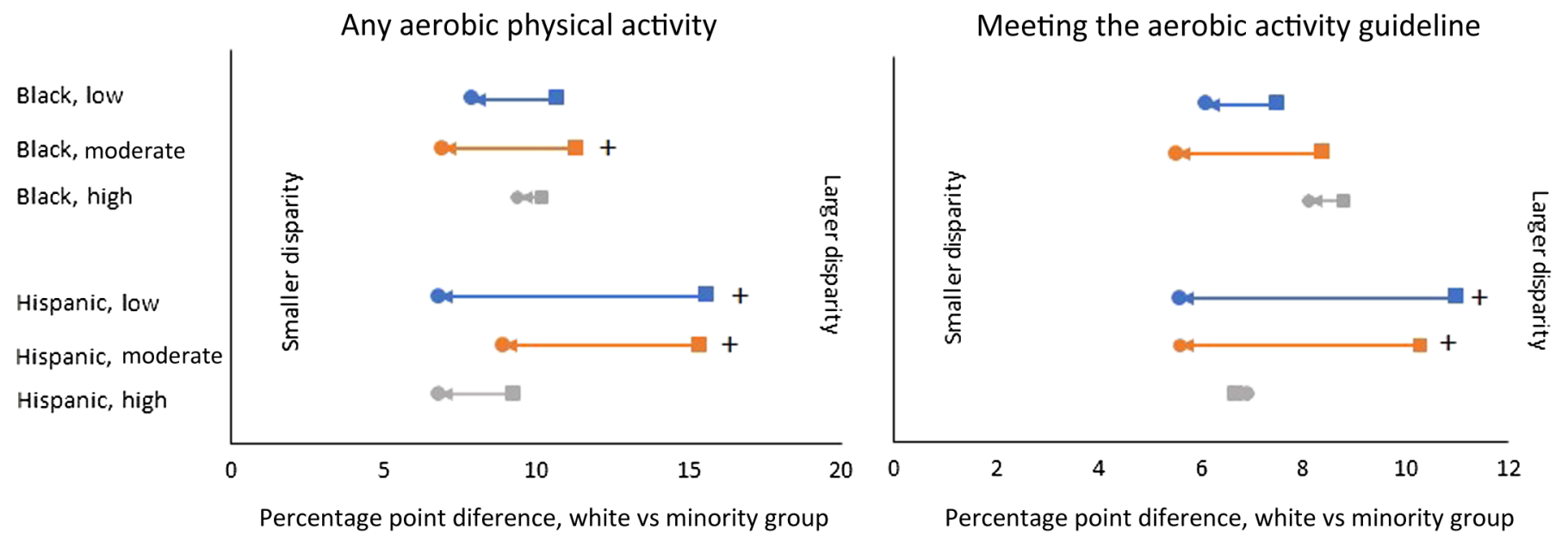

Figure 6 - Engaging in any aerobic activity and meeting the aerobic activity guideline estimates for the differences between non-Hispanic white and non-Hispanic black (black) and non-Hispanic white and Hispanic (Hispanic) adults at low, moderate, and high income levels. Differences between white adults and members of racial/ethnic minority groups from 1998-2000 to 2016-2018. Square indicates difference between white and racial/ethnic minority groups in 1998-2000. Circle indicates difference between white and racial/ethnic minority groups in 2016-2018. Plus indicates significant narrowing in difference (D1 - D2 > 0; [1998-2000 white - minority] - [2016-2018 white - minority] > 0). D1 indicates the difference in prevalence at the first time period (1998-2000); D2, difference in prevalence at the last time period (2016-2018).

Meeting the muscle-strengthening guideline

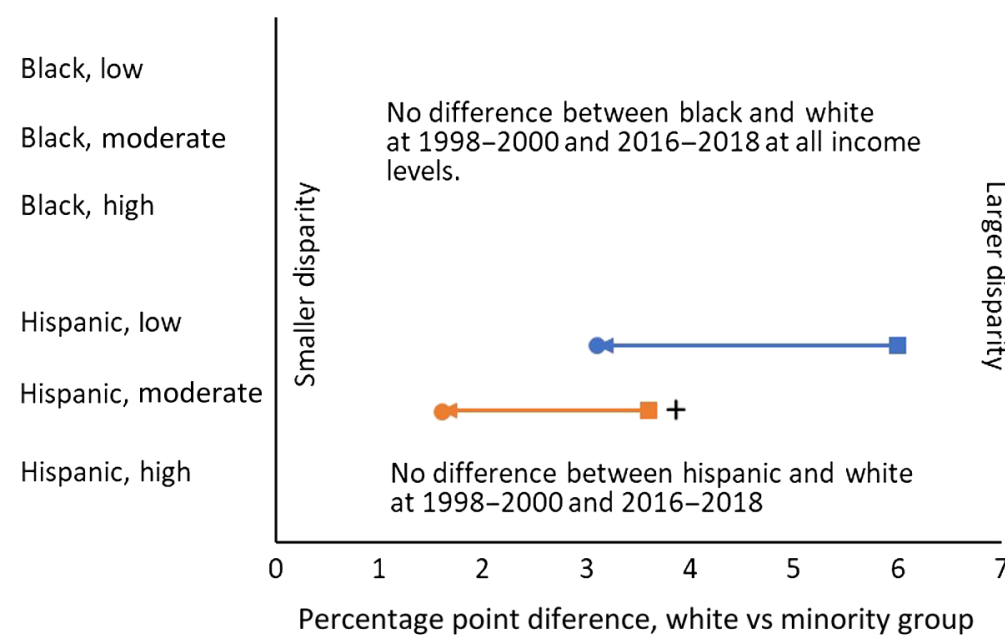

Meeting the combined aerobic activity and muscle-strengthening guideline

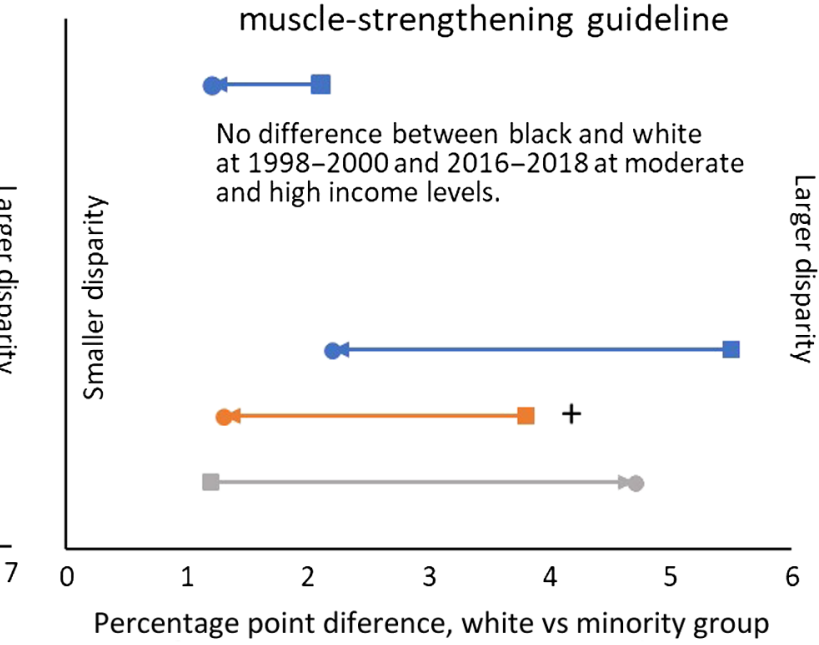

Figure 7 - Muscle-strengthening guideline and combined guidelines estimates for the differences between non-Hispanic white and non-Hispanic black and non-Hispanic white and Hispanic (Hispanic) adults at low, moderate, and high income levels. Text, not lines, is used where there is no difference between white adults and members of racial/ethnic minority groups from 1998-2000 to 2016-2018. Square indicates difference between white and racial/ ethnicity minority groups in 1998-2000. Circle indicates difference between white and racial/ethnic minority groups in 2016-2018. Plus indicates significant narrowing in difference (D1 - D2 >0; [1998-2000 white - minority] - [2016-2018 white - minority] $>0$ ). D1 indicates the difference in prevalence at the first time period (1998-2000); D2, difference in prevalence at the last time period (2016-2018).

participation across racial/ethnic groups and income levels reported across various national surveillance systems is encouraging. Our study also adds to the literature because of its inclusion of income as a moderating variable on the association between race/ethnicity and physical activity participation.

Our study found that racial/ethnic disparities in aerobic physical activity participation persisted across all income levels. Several factors can contribute to these disparities, including key social determinants of health, such as the environments where people live, work, and play.
Communities with more residents who are members of racial/ethnic minority groups and who have lower incomes may lack access to opportunities to be physically active, in part, because of long-standing, systemic health and social inequities. ${ }^{23,24}$ For example, these communities may lack features that support walking and have limited access to well-maintained or safe parks. ${ }^{24}$ Community strategies designed to promote physical activity ${ }^{25}$ that are tailored to lowincome, racial/ethnic minority groups can help ensure equitable access to supports and opportunities for physical activity. 
These strategies are part of efforts by the Racial and Ethnic Approaches to Community Health (REACH) program of the Centers for Disease Control and Prevention. ${ }^{26} \mathrm{REACH}$ focuses on reducing chronic diseases for multiple racial and ethnic groups (African Americans, Hispanics, American Indians/Alaska Natives, Asian Americans, and Native Hawaiians/Other Pacific Islanders) in communities with high rates of chronic diseases by promoting healthy behaviors and chronic disease management. ${ }^{26}$ Communities funded by REACH use several strategies, including one specifically designed to improve physical activity through transportation and land-use interventions that connect activity-friendly routes (eg, sidewalks, bicycle paths, paths, public transit) to everyday destinations (eg, homes, early care and education facilities, schools, worksites, parks, recreation centers). ${ }^{26,27}$ This strategy aligns with goals outlined in The Guide to Community Preventive Services ${ }^{25}$ and Step It Up! The Surgeon General's Call to Action to Promote Walking and Walkable Communities ${ }^{28}$ to increase access to opportunities to be physically active. A $\mathrm{REACH}$ recipient in South Los Angeles, California, used zoning and land-use strategies to make it easier for community members to safely engage in physical activity in open spaces, parks, and recreational facilities. The community also plans to improve access to local walking paths, biking paths, and other sustainable modes of transportation.

Physical activity promotion is also part of REACH's strategy to improve links between community programs and clinical services for priority populations. Activities include encouraging clinicians to refer patients to community health programs designed to improve a variety of health outcomes and behaviors, including physical activity. ${ }^{26,27}$ For example, in Birmingham, Alabama, patients have been prescribed physical activity through the Parks Rx program, which links patients through local health care systems to online resources to help them find local parks where they can be active. $^{29}$

Other efforts designed to make access to physical activity more equitable and inclusive include the Active People, Healthy Nation $^{\text {SM }}$ initiative, which was launched by Centers for Disease Control and Prevention in 2018. This national initiative seeks to help 27 million Americans be more physically active by 2027 as a way to improve their health and quality of life. ${ }^{30,31}$ It promotes strategies recommend by the Community Services Task Force to increase physical activity. One of these strategies is social support, which has been shown to help people start, maintain, or increase physical activity through group activities, such as buddy systems and activity clubs. ${ }^{32}$ For example, GirlTrek, an organization that promotes pride, independence, and social support for African American women, sponsors regular walking sessions. ${ }^{33}$ GirlTrek started in 1996 with 2 friends and, in 2016, became the largest health nonprofit for black women and girls in the United States. ${ }^{33}$ Community-based efforts that are tailored to the needs of specific populations, such as those supported by REACH and Active People, Healthy Nation, can help eliminate disparities in physical activity participation between different racial/ethnic groups.

\section{Limitations}

This study has several limitations. First, NHIS assesses selfreported physical activity, which is subject to recall and social desirability biases. ${ }^{34}$ Second, the NHIS physical activity questions ask adults to report on physical activity that occurred in bouts of $\geq 10$ minutes. Although the 2008 Physical Activity Guidelines for Americans included a bout minimum requirement of at least 10 minutes for the adult aerobic physical activity guideline, this requirement was removed from the second edition of the guidelines, released in 2018. ${ }^{2,35}$ Recent evidence suggests that elimination of the 10-minute bout minimum may have little effect on physical activity estimates. ${ }^{36}$ Finally, NHIS asks adults to report only on their LTPA, not physical activity performed in other domains (eg, occupational, household, transportation). Increasing linear trends in LTPA participation appear consistent across racial/ ethnic groups and educational attainment, but it is unknown whether the inclusion of aerobic physical activity in additional domains would affect the findings reported here. ${ }^{37}$ The strength of this study was the use of data from a large, nationally representative sample of US adults, which allowed for the examination of racial/ ethnic group and income levels simultaneously.

\section{Conclusions}

Our findings suggest that LTPA levels have increased over time across all racial/ethnic groups at all income levels and the prevalence of meeting the aerobic guideline is substantially higher than meeting the muscle-strengthening guideline. Aerobic physical activity participation remains lower among black and Hispanic adults compared with white adults across all income levels, although differences have narrowed for some subgroups. Racial and ethnic disparities in meeting the muscle-strengthening and combined guidelines were less commonly observed. Strategies to increase both aerobic and muscle-strengthening activity that are tailored to specific racial/ethnic groups, particularly those in lowincome areas, may help continue progress in reducing disparities.

\section{Acknowledgment}

The findings and conclusions in this manuscript are those of the authors and do not necessarily represent the official position of the Centers for Disease Control and Prevention.

\section{References}

1. 2018 Physical Activity Guidelines Advisory Committee. 2018 Physical Activity Guidelines Advisory Committee Scientific Report. Washington, DC: U.S. Department of Health and Human Services; 2018.

2. United States Department of Health and Human Services (HHS). Physical Activity Guidelines for Americans. 2nd ed. Washington, DC: USDHHS; 2018.

3. Hyde ET, Whitfield GP, Omura JD, et al. Trends in meeting the physical activity guidelines: muscle-strengthening alone and combined with aerobic activity, United States, 1998-2018. J Phys Act Health. 2021;18(suppl):S37-S44. doi:10.1123/jpah.2021-0077

4. Centers for Disease Control and Prevention. Behavioral risk factor surveillance system. 2020. https://www.cdc.gov/brfss/index.html. Accessed February 20, 2021.

5. Centers for Disease Control and Prevention. National health and nutrition examination survey. 2021. https://www.cdc.gov/nchs/ nhanes/index.htm. Accessed February 20, 2021.

6. Whitfield GP, Ussery EN, Saint-Maurice PF, Carlson SA. Trends in aerobic physical activity participation across multiple domains among US Adults, National Health and Nutrition Examination Survey 2007/ 2008 to 2017/2018. J Phys Act Health. 2021;18(suppl):513-518. doi:10.1123/jpah.2021-0173. 
7. Division of Nutrition Physical Activity and Obesity. Nutrition, physical activity, and obesity: data, trends and maps. 2021. https:// www.cdc.gov/nccdphp/dnpao/data-trends-maps/index.html. Accessed January 15, 2021.

8. Moore LV, Harris CD, Carlson SA, Kruger J, Fulton JE. Trends in no leisure-time physical activity-United States, 1988-2010. Res $Q$ Exerc Sport. 2012;83(4):587-591. PubMed ID: 23367822 doi:10. 1080/02701367.2012.10599884. Accessed October 25, 2019.

9. Villarroel MA, Blackwell DL, Jen A. Tables of summary health statistics for US adults: 2018 National Health Interview Survey. National Center for Health Statistics. 2019. https://ftp.cdc.gov/pub/ Health_Statistics/NCHS/NHIS/SHS/2018_SHS_Table_A-14.pdf. Accessed October 25, 2019.

10. Crook ED, Peters M. Health disparities in chronic diseases: where the money is. Am J Med Sci. 2008;335(4):266-270. PubMed ID: 18461728 doi:10.1097/MAJ.0b013e31816902f1

11. Falci L, Shi Z, Greenlee H. Multiple chronic conditions and use of complementary and alternative medicine among US Adults: results from the 2012 National Health Interview Survey. Prev Chronic Dis. 2016;13:E61. PubMed ID: 27149072 doi:10.5888/pcd13.150501

12. Marmot M, Friel S, Bell R, Houweling TA, Taylor S. Closing the gap in a generation: health equity through action on the social determinants of health. Lancet. 2008;372(9650):1661-1669. doi:10.1016/ S0140-6736(08)61690-6

13. Whitfield GP, Carlson SA, Ussery EN, et al. Racial and ethnic differences in perceived safety barriers to walking, United States National Health Interview Survey-2015. Prev Med. 2018;114:5763. doi:10.1016/j.ypmed.2018.06.003

14. LaVeist TA. Disentangling race and socioeconomic status: a key to understanding health inequalities. J Urban Health. 2005;82(2)(suppl 3):26-34. doi:10.1093/jurban/jti061

15. United States Department of Health and Human Services (HHS). Healthy People 2020. physical activity: objectives. 2014. http:// www.healthypeople.gov/2020/topics-objectives/topic/physical-activity/ objectives. Accessed October 25, 2019.

16. Eugeni ML, Baxter M, Mama SK, Lee RE. Disconnections of African American public housing residents: connections to physical activity, dietary habits and obesity. Am J Community Psychol. 2011;47(3-4):264-276. PubMed ID: 21181553 doi:10.1007/ s10464-010-9402-1

17. Whitfield GP, Ussery EN, Saint-Maurice PF, Carlson SA. Trends in aerobic physical activity participation across multiple domains among US Adults, National Health and Nutrition Examination Survey 2007/ 2008 to 2017/2018. J Phys Act Health. 2021;18(suppl):S64-S73. doi:10.1123/jpah.2021-0173

18. United States Department of Health and Human Services (HHS). Healthy People 2030. Physical activity: objectives. 2021. https:// health.gov/healthypeople/objectives-and-data/browse-objectives/ physical-activity. Accessed February 25, 2021.

19. National Center for Health Statistics. National Health Interview Survey. Public-use data file and documentation. 2018. https://www.cdc. gov/nchs/nhis/1997-2018.htm. Accessed August 20, 2020.

20. Center on Budget and Policy Priorities. A quick guide to SNAP eligibility and benefits. 2020. https://www.cbpp.org/research/foodassistance/a-quick-guide-to-snap-eligibility-and-benefits. Accessed March 26, 2021.

21. ObamaCareFacts.com. Federal poverty level guidelines. 2021. https:// obamacarefacts.com/federal-poverty-level/. Accessed March 26, 2021.

22. National Health Interview Survey. 2018. Public-use data file and documentation (2018). ftp://ftp.cdc.gov/pub/Health_Statistics/NCHS/
Dataset_Documentation/NHIS/2018/srvydesc.pdf. Accessed February 25, 2021.

23. Centers for Disease Control and Prevention. Health equity considerations and racial and ethnic minority groups. 2021. https://www.cdc. gov/coronavirus/2019-ncov/community/health-equity/race-ethnicity. html. Accessed January 22, 2021.

24. Taylor W. Do all children have places to be active? Disparities in access to physical activity environments in racial and ethnic minority and lower-income communities. A research synthesis. 2011. http:// activelivingresearch.org/do-all-children-have-places-be-activedisparities-access-physical-activity-environments-racial-and. Accessed October 20, 2020.

25. Community Preventive Services Task Force. Physical activity: built environment approaches combining transportation system interventions with land use and environmental design. 2016. https://www. thecommunityguide.org/sites/default/files/assets/PA-BuiltEnvironments.pdf. Accessed October 29, 2020.

26. REACH. CDC's Racial and Ethnic Approaches to Community Health Program. Centers for Disease Control and Prevention. 2020. https:// www.cdc.gov/chronicdisease/resources/publications/factsheets/reach. htm. Accessed February 22, 2021.

27. REACH. 2014. https://www.cdc.gov/nccdphp/dnpao/state-localprograms/reach/2014/index.html. Accessed March 15, 2021.

28. U.S. Department of Health and Human Services. Step It Up! The Surgeon General's Call to Action to Promote Walking and Walkable Communities. U.S. Department of Health and Human Services, Office of the Surgeon General; 2015.

29. Alabama providers prescribe physical activity via the Parks $R x$ program. 2017. https://nccd.cdc.gov/nccdsuccessstories/Template Three.aspx?s=13938\&ds=1. Accessed February 25, 2021.

30. Fulton JE, Buchner DM, Carlson SA, et al. CDC's active people, healthy nation(SM): creating an active America, together. J Phys Act Health. 2018;15(7):469-473. PubMed ID: 29932005 doi:10.1123/ jpah.2018-0249

31. Schmid TL, Fulton JE, McMahon JM, Devlin HM, Rose KM, Petersen R. Delivering physical activity strategies that work: active people, healthy nation SM. J Phys Act Health. 2021;18(4):352-356. PubMed ID: 33639612 doi:10.1123/jpah.2020-0656

32. Kahn EB, Ramsey LT, Brownson RC, et al. The effectiveness of interventions to increase physical activity. A systematic review. Am J Prev Med. 2002;22(4):73-107. doi:10.1016/S0749-3797(02)00434-8

33. GirlTrek. GirlTrek. https://www.girltrek.org/. Accessed March 15, 2021.

34. Rzewnicki R, Vanden Auweele Y, De Bourdeaudhuij I. Addressing overreporting on the International Physical Activity Questionnaire (IPAQ) telephone survey with a population sample. Public Health Nutr. 2003;6(3):299-305. PubMed ID: 12740079 doi:10.1079/ PHN2002427

35. United States Department of Health and Human Services (HHS). 2008 Physical Activity Guidelines for Americans. 2008. https:// health.gov/sites/default/files/2019-09/paguide.pdf. Accessed October 20, 2020.

36. Ussery EN, Watson KB, Carlson SA. The influence of removing the ten-minute bout requirement on national physical activity estimates. Prev Chronic Dis. 2020;17:E19. PubMed ID: 32105588 doi:10.5888/ pcd17.190321

37. Whitfield GP, Ussery EN, Carlson SA. Combining data from assessments of leisure, occupational, household, and transportation physical activity among US Adults, NHANES 2011-2016. Prev Chronic Dis. 2020;17:E117. doi:10.5888/pcd17.200137 